Applied Optics, 2005, 44(13): 2519-2529

\title{
Crossed source-detector geometry for a novel spray diagnostic: Monte Carlo simulation and analytical results
}

\author{
Edouard Berrocal, Dmitry Y. Churmakov, Vadim P. Romanov, \\ Mark C. Jermy, and Igor V. Meglinski
}

\begin{abstract}
Sprays and other industrially relevant turbid media can be quantitatively characterized by light scattering. However, current optical diagnostic techniques generate errors in the intermediate scattering regime where the average number of light scattering is too great for the single scattering to be assumed, but too few for the diffusion approximation to be applied. Within this transitional single-to-multiple scattering regime, we consider a novel crossed source-detector geometry that allows the intensity of single scattering to be measured separately from the higher scattering orders. We verify Monte Carlo calculations that include the imperfections of the experiment against analytical results. We show quantitatively the influence of the detector numerical aperture and the angle between the source and the detector on the relative intensity of the scattering orders in the intermediate single-to-multiple scattering regime. Monte Carlo and analytical calculations of double light-scattering intensity are made with small particles that exhibit isotropic scattering. The agreement between Monte Carlo and analytical techniques validates use of the Monte Carlo approach in the intermediate scattering regime. Monte Carlo calculations are then performed for typical parameters of sprays and aerosols with anisotropic (Mie) scattering in the intermediate single-to-multiple scattering regime.
\end{abstract}

\section{Introduction}

The scattering of light by disperse randomly inhomogeneous scattering media is important in many applications including optical diagnostics in meteorology, powder industries and sprays for combustors, firefighting, drug delivery, and biomedicine. In all these applications, knowledge and measurements of optical parameters of disperse medium are required. Typical parameters measured are the mean free transport path length, shape and size of the scattering particles, and their concentration within the medium. If the scattering power of the medium is low (mean number of scattering events is $\leq 3$ ), these parameters can be recovered from the intensity of single-scattered radiation by assuming that only single scattering takes place. ${ }^{1-3}$ However, if the

E. Berrocal (e.berrocal.2002@cranfield.ac.uk), D. Y. Churmakov, M. C. Jermy, and I. V. Meglinski are with the School of Engineering, Cranfield University, Cranfield MK43 0AL, UK. V. P. Romanov is with the Department of Physics, St. Petersburg State University, Petrodvoretz 198504, Russia. medium is turbid (mean number of scattering events is $>3-6$ ), the intensity due to high orders of scattering dominates that due to single scattering at the detector, and the single-scattering assumption becomes invalid. Nevertheless, the average parameters of such a medium can be obtained by the diffusion approximation. ${ }^{4,5}$

Greater complications arise in the intermediate case (mean number of scattering events is $\sim 3-6$ ), where the average number of scattering events is too high for single scattering to be assumed, but too low for the diffusion approximation to be applied. This situation is typical for many practical applications, including industrial sprays. It should be pointed out, however, that, in the field of sprays and aerosols, most currently available optical diagnostic instruments are designed to operate in media of low scattering power with the single-scattering assumption. The development of instruments operating in the intermediate scattering regime is required. The intermediate scattering regime is encountered when measurements are made near the nozzle (as required for boundary-condition data for computational fluid dynamics calculations of spray systems or to validate liquid breakup models) or when the path length of the light in the spray is long (as in the case of high-flowrate mixing systems such as fuel atomizers for large 
combustors). To develop such instruments and to characterize their accuracy, an estimation of the main scattering orders is required. It is necessary to have a computing photon-tracking technique validated in this intermediate regime.

For dilute intermediate scattering media the parameters of scattering particles can be obtained from the angular dependence of the scattered light intensity, from attenuation of light due scattering and absorption, or by interference and diffraction effects. Attenuation methods can be applied not only to small particles but also to large particles, i.e., with diameters greater than the incident laser beam diameter. ${ }^{6,7}$ The principal advantages of diffraction methods are their good repeatability and the detailed information they give on the particle size distribution. ${ }^{8,9}$ For angular dependence methods, one of the most efficient approaches is multiwavelength measurement. ${ }^{10-12}$ More recently, polarization methods have become common. ${ }^{13}$ In addition there are methods based on the comparative analysis of both elastic scattering and fluorescence arising from fluorescent dyes mixed into the particles. ${ }^{14}$

The transport of light in such turbid media can be predicted by the solution of the radiation transport equation coupled with an appropriate scattering theory, e.g., Mie ${ }^{1}$ or Rayleigh-Gans scattering. ${ }^{2}$ However, because of the complex geometries and range of particle sizes encountered in practical applications, it is rarely possible to find an analytical solution. Instead numerical solutions are used. One of the most popular and versatile is the Monte Carlo (MC) approach. ${ }^{15-17} \mathrm{MC}$ photon transport simulation is well established in biomedical applications, ${ }^{18,19}$ astronomical and meteorological applications, ${ }^{17,20-24}$ and more recently in industrial sprays. ${ }^{25-27}$ Recently, with a combination of the stochastic MC technique and an iterative solution of the Bethe-Salpeter equation, it has been shown that the simulation of the optical path of a photon packet undergoing an $n$th scattering event directly corresponds to the $n$ th-order ladder diagram contribution. ${ }^{28,29} \mathrm{MC}$ simulations usually describe only the trajectory of the scattered radiation, but polarization changes inside and outside of the medium can also be taken into account based on the Stokes-Muller formalism, ${ }^{30-32}$ the Jones formalism, ${ }^{33}$ and the Rayleigh-Debye scattering theory. ${ }^{34-36}$

The MC simulation allows for the quantification of the effect of differences between ideal theoretical geometries and experiments with their imperfections (e.g., imperfectly monodisperse particles, divergence of a laser beam, finite apertures). It is important, however, to verify the operation of any simulation code by comparison with theory and experimental results. In this paper we compare analytical calculations of scalar intensity for the double light scattering with the results of MC simulation. The comparison serves two purposes. First, it verifies the MC code against the analytical solution. Second, it allows the analytical solution to be tested by indirect comparison with (imperfect) the experimental results

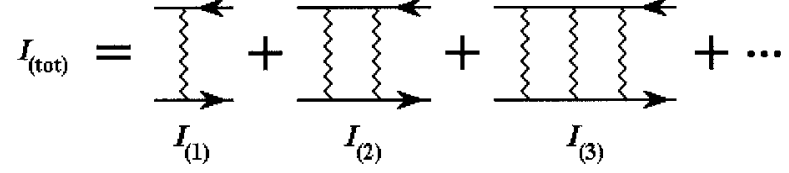

Fig. 1. Schematic presentation of the scattering intensity as a series of ladder diagrams. ${ }^{5,40-42}$

through the MC results. Similar MC and analytical comparisons have been carried out for detection geometries other than the one presented here to support the $\mathrm{MC}$ investigation of meteorological ${ }^{37}$ colloidal suspension ${ }^{38}$ and biomedical ${ }^{39}$ problems. This paper is organized as follows. In Section 2 the theoretical aspects of the scattering problem are explained, and general analytical formulas for the intensity of different scattering orders are derived. The design of the $\mathrm{MC}$ code and descriptions of the simulation are considered in Section 3. The results and discussions are presented in Section 4, and some remarks and conclusions are given in Section 5. Appen$\operatorname{dix} \mathrm{A}$ is a list of the nomenclature used in the paper.

\section{Analytical Description of Low Scattering Orders}

The intensity of scattering of optical radiation propagated in a randomly inhomogeneous scattering medium can be presented as an infinite series of scattering orders,, $50-42$ usually illustrated by ladder diagrams (Fig. 1). The convergence of this series depends on the mean square of permittivity fluctuations and the characteristic size of the scattering medium.

In the case of low scattering power, the series of scattering orders is reduced to the first term describing single scattering. All other terms of the series are neglected or considered as a perturbation to this single-scattering term. In the case of multiple scattering, it is assumed that all the terms of the series have same order of magnitude intensity, and the diffusion approximation is applied.5,40

In the intermediate scattering regime, both singleand high-order terms of the series should be considered. To separate these scattering terms from each other we apply an idea originally suggested in the study of critical phenomena and second-order phase transition. ${ }^{42}$ Schematically this idea is represented in Fig. 2. The laser source $S$ illuminates the medium with a thin cylindrical laser beam. Detector $D$ is confined by a series of small aperture diaphragms so that the detected radiation is localized in a cylindrical volume $V_{2}$, which is equal to the volume filled by the incident laser beam $V_{1}$ (see Fig. 2). Thus, if $V_{1}$ and $V_{2}$ do not intersect, photons must experience at least two scattering events to be detected. Similarly, for singly scattered photons to reach the detector, the volumes must intersect, i.e., $h$ must be less than the diameter of the cylinder (see Fig. 2). When the characteristic diameter of the scattering volume $V$ is much less than the distance to the observation point, the intensity of single-scattering light takes the form ${ }^{5,41,42}$ 


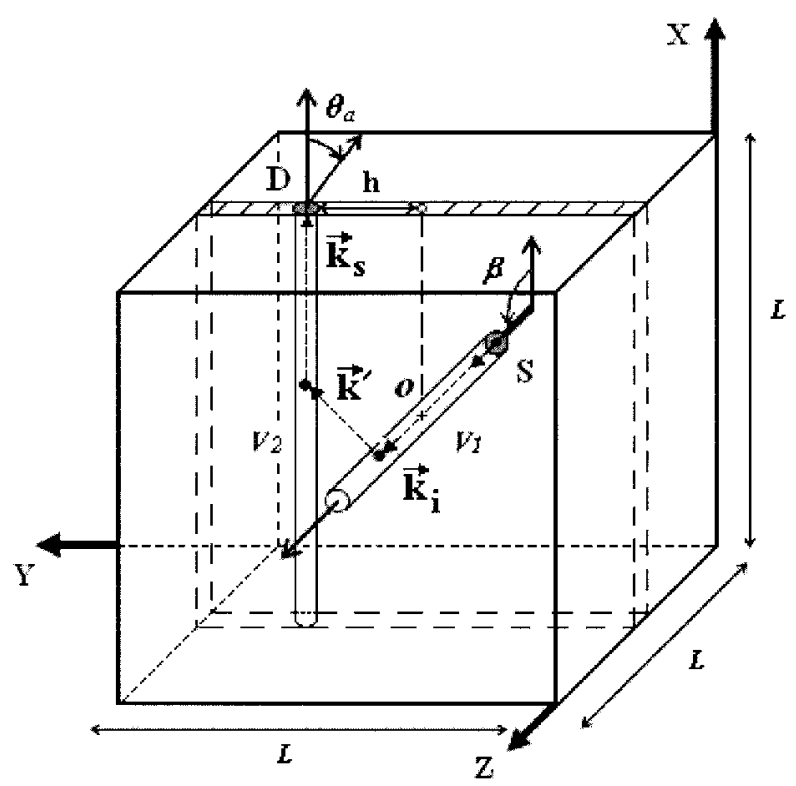

Fig. 2. Computational/proposed experimental geometry. The laser source $S$ and detector $D$ sample two cylindrical volumes $V_{1}$ and $V_{2}$, within which single scattering occurs at points $\mathbf{r}_{1}$ and $\mathbf{r}_{2}$. If the volumes are separated by distance $h$, the detector collects only double and higher orders of scattering. $\mathbf{k}_{i}, \mathbf{k}^{\prime}$, and $\mathbf{k}_{s}$ are the wave vectors of the incident, intermediate, and detected doublescattered light, respectively. The scattering volume is a cube of $L=50 \mathrm{~mm}$ defined in a three-dimensional coordinate system. One of the corners of the cube corresponds to the origin of the $(X, Y, Z)$ frame.

$$
I_{(1)}=\frac{I_{0} V}{r^{2}} \frac{k_{0}{ }^{4}}{(4 \pi)^{2}}\left(\delta_{\alpha \beta}-\frac{k_{s \alpha} k_{s \beta}}{k^{2}}\right)^{2} G(\mathbf{q}) \exp \left[-\mu_{t}\left(l_{1}+l_{2}\right)\right] .
$$

Here $I_{0}$ is the intensity of the incident light, $V$ is the scattering volume, $r$ is the distance to the observation point, $k_{0}=\omega / c$, where $\omega$ is the cycle frequency and $c$ is the speed of light in vacuum. The exponential multiplier describes attenuation along a path $l_{1}$ before and a path $l_{2}$ after the scattering event. $\mu_{t}$ is the extinction coefficient, describing the attenuation of light due to elastic and inelastic interactions of laser radiation within the medium: $\mu_{t}=\mu_{s}+\mu_{a}$, where $\mu_{s}$ and $\mu_{a}$ are the scattering and absorption coefficients, respectively; and $\mathbf{q}=\mathbf{k}_{s}-\mathbf{k}_{i}$ is the scattering wave vector. Vectors $\mathbf{k}_{s}$ and $\mathbf{k}_{i}$ are the wave vectors of the incident and scattered light, respectively; and $\mathbf{k}$ can be expressed as a vector $k(\mathbf{r} / f)$, where $\mathbf{r} / r$ is the direction toward an observation point. A factor $\left[\delta_{\alpha \beta}\right.$ $\left.-\left(k_{s \alpha} k_{s \beta} / k^{2}\right)\right]^{2}$ accounts for the transverse nature of the scattered electromagnetic wave. $\alpha$ and $\beta$ are the indices of polarization of the incident and scattered light, respectively. $G(\mathbf{q})$ is the correlation function of the permittivity fluctuations, described for a weakly scattering medium of spherical scattering particles by the approximate formula ${ }^{5,41,42}$ :

$$
G(\mathbf{q}) \approx\left(\frac{2 \pi \Delta \varepsilon}{3}\right)^{2} a^{6} N
$$

Here $N$ is the particle density number, $\Delta \varepsilon$ is the difference of permittivities of particles and the host medium, and $a$ is the particle diameter.

The intensity of double scattering is described as

$$
\begin{aligned}
I_{(2)}= & \frac{I_{0} k_{0}^{8}}{r^{2}(4 \pi)^{4}} \int_{V_{1}} \mathrm{~d} \mathbf{r}_{1} \int_{V_{2}} \mathrm{~d} \mathbf{r}_{2} F_{\alpha \beta}\left(\mathbf{k}_{s}, \mathbf{k}_{i}, \mathbf{k}^{\prime}\right) \frac{1}{\left|\mathbf{r}_{2}-\mathbf{r}_{1}\right|^{2}} \\
& \times G\left(\mathbf{k}^{\prime}-\mathbf{k}_{i}\right) G\left(\mathbf{k}_{s}-\mathbf{k}^{\prime}\right) \exp \left[-\mu_{t}\left(l_{1}+l_{2}+\mid \mathbf{r}_{2}\right.\right. \\
& \left.\left.-\mathbf{r}_{1} \mid\right)\right],
\end{aligned}
$$

where

$$
\begin{aligned}
F_{\alpha \beta}\left(\mathbf{k}_{s}, \mathbf{k}_{i}, \mathbf{k}^{\prime}\right)= & \left(\delta_{\alpha \eta}-\frac{k_{\mathrm{i} \alpha}{ }^{\prime} k_{\mathrm{i \eta}}{ }^{\prime}}{k^{2}}\right)\left(\delta_{\alpha v}-\frac{k_{\mathrm{i} \alpha}{ }^{\prime} k_{\mathrm{i} v}{ }^{\prime}}{k^{2}}\right) \\
& \times\left(\delta_{\beta \eta}-\frac{k_{s \beta} k_{s \eta}}{k^{2}}\right)\left(\delta_{\beta v}-\frac{k_{\mathrm{s} \beta} k_{\mathrm{sv}}}{k^{2}}\right)
\end{aligned}
$$

is the polarization factor, $V_{1}$ is the illuminated volume, and the scattered light is collected by the detector from the volume $V_{2}$. Summation occurs over the indices (except $\alpha$ and $\beta$ ) where a quantity has more than one index. Equation (3) describes the intensity of doubly scattered light reaching the detector, i.e., singly scattered light entering volume $V_{2}$ and being scattered into the $\mathbf{k}_{s}$ direction with a wave vector $\mathbf{k}_{s}$ $-\mathbf{k}^{\prime}$. Note that all first-order scattering events occur in the volume $V_{1}$ with a wave vector $\mathbf{k}^{\prime}-\mathbf{k}_{i}$, where $\mathbf{k}^{\prime}$ is the intermediate wave vector (see Fig. 2).

To validate the $\mathrm{MC}$ technique and to avoid the complex calculation, let us consider the case of scalar scattering with the source-detector geometry represented in Fig. 2. The details of the polarization changes within the medium ${ }^{30-36}$ are not considered in this paper, but will be reported in the future.

In this case the intensities of single and double light scatterings can be described as

$$
\begin{aligned}
I_{(1)}= & \frac{I_{0} V}{r^{2}} k_{0}^{4}\left(\frac{\Delta \varepsilon}{6}\right)^{2} a^{6} N \exp \left[-\mu_{t}\left(l_{1}+l_{2}\right)\right], \\
I_{(2)}= & \frac{I_{0} k_{0}^{8}}{r^{2}}\left(\frac{\Delta \varepsilon}{6}\right)^{4} \int_{V_{1}} \mathrm{~d} \mathbf{r}_{1} \int_{V_{2}} \mathrm{~d} \mathbf{r}_{2} \frac{1}{\left|\mathbf{r}_{2}-\mathbf{r}_{1}\right|^{2}} \frac{k_{y}{ }^{2}}{k^{2}} \\
& \times\left[1-\frac{k_{y}{ }^{2}}{k^{2}}-\frac{\left(\mathbf{k}_{s} \mathbf{k}^{\prime}\right)^{2}}{k^{4}}\right] a^{12} N^{2} \\
& \times \exp \left[-\mu_{t}\left(l_{1}+l_{2}+\left|\mathbf{r}_{2}-\mathbf{r}_{1}\right|\right)\right],
\end{aligned}
$$

respectively. It is easy to see that the ratio of $I_{(2)}$ to $I_{(1)}$ depends on a geometric term, which can be evaluated if the geometry is known, and on the factor $A$ $\sim(\Delta \varepsilon)^{2} a^{6} N$ describing optical properties of the medium. Thus the ratio of measured $I_{(2)}$ and $I_{(1)}$ gives the values of the medium optical parameters. However, to make the results obtained more reliable, higher orders of scattering should be taken into account. The evaluation of analytical expressions for the high scat- 
tering orders $I_{(3)}, I_{(4)}, I_{(5)}$, and so on is an extraordinary complex mathematical problem requiring the calculation of multifold integrals. The double-scattering intensity calculation needs cumbersome computation of the sixfold integral [Eq. (7)], whereas $I_{(3)}$ requires calculation of the ninefold integral, $I_{(4)}$ of the 12 -fold integral, and so on.

$$
\begin{aligned}
I_{(2)}= & A \int_{-L_{1 / 2}}^{L_{1 / 2}} \mathrm{~d} l_{1} \int_{-L_{2 / 2}}^{L_{2 / 2}} \mathrm{~d} l_{2} \int_{0}^{R_{1}} r_{1} \mathrm{~d} r_{1} \int_{0}^{R_{2}} r_{2} \mathrm{~d} r_{2} \\
& \times \int_{0}^{2 \pi} \mathrm{d} \phi_{1} \int_{0}^{2 \pi} \mathrm{d} \phi_{2} \frac{1}{\left|\mathbf{r}_{2}-\mathbf{r}_{1}\right|^{2}} \\
& \times \exp \left[-\mu_{t}\left(l_{1}+l_{2}+\left|\mathbf{r}_{2}-\mathbf{r}_{1}\right|\right)\right],
\end{aligned}
$$

where $L_{1}, L_{2}$ are the lengths and $R_{1}, R_{2}$ are the radii of the $V_{1}$ and $V_{2}$ cylinders directed along the axes $Z$ and $X$, respectively (see Fig. 2).

To avoid this complex calculation, the numerical MC technique is applied to calculate the higher-order scattering terms.

\section{Monte Carlo Simulation}

The exact implementation of the MC technique for calculation of scattering light intensity in an inhomogeneous scattering medium depends on the application. ${ }^{18-30}$ The MC scheme presented here is suitable for the geometry described in Fig. 2 and possesses the following characteristics. The scattering medium is defined by a cube of $L=L_{1}=L_{2}$ $=50 \mathrm{~mm}$ in a three-dimensional coordinate system. The medium is assumed nonabsorbing $\left(\mu_{a}=0\right)$ and homogeneous. The source $S$ is defined by a cylindrical laser beam $1 \mathrm{~mm}$ in diameter (i.e., $R_{1}=R_{2}$ $=0.5 \mathrm{~mm}$ ), which enters through a face of the sampling cube with an angle $\beta$ corresponding to the source-detector angle. This angle $\beta$ ranges between $0^{\circ}$ (forward-scattering detection) and $180^{\circ}$ (backscattering detection) so that the laser beam always passes through the central point $O$ of the cube, illuminating a cylindrical volume $V_{1}$ through the scattering medium (Fig. 2). The detector $D$ is represented by an aperture $1 \mathrm{~mm}$ in diameter located on the top face of the cube and positioned at different distances $h$ from the central vertical axis of the cube. The scattering coefficient $\mu_{s}$ ranges between 0.04 and $0.18 \mathrm{~mm}^{-1}$. This range allows the transition from single to multiple scattering to be investigated in the given geometry.

MC modeling of the photon trajectories within the medium consists of the following steps. The path length of a photon packet between two scattering events is given by ${ }^{15}$

$$
l_{i}=\frac{\ln (\xi)}{\mu_{s}}
$$

where $\xi$ is a random number uniformly distributed between 0 and 1 . To specify the position of a photon and its direction of propagation, the absolute and local coordinate systems are used. After scattering, the new direction is specified by the polar and azimuth angles, i.e., by $\theta_{s}$ and $\varphi$, respectively.

The directions of the photon packet propagation before and after each scattering event are defined by unit vectors $\mathbf{u}_{i}$ and $\mathbf{u}_{s}$, with $\mathbf{u}_{i}$ and $\mathbf{u}_{s}$ being collinear to vectors $\mathbf{k}_{i}$ and $\mathbf{k}_{s}$, respectively. The following transformation relates them:

$$
\begin{aligned}
{\left[\begin{array}{l}
u_{s x} \\
u_{s y} \\
u_{s z}
\end{array}\right]=} & {\left[\begin{array}{lll}
\frac{1}{\left(1-u_{i z}{ }^{2}\right)^{1 / 2}} u_{i x} u_{i z} & -\frac{1}{\left(1-u_{i z}{ }^{2}\right)^{1 / 2}} u_{i y} & u_{i x} \\
\frac{1}{\left(1-u_{i z}{ }^{2}\right)^{1 / 2}} u_{i y} u_{i z} & \frac{1}{\left(1-u_{i z}{ }^{2}\right)^{1 / 2}} u_{i x} & u_{i y} \\
-\left(1-u_{i z}{ }^{2}\right)^{1 / 2} & 0 & u_{i z}
\end{array}\right] } \\
& \times\left[\begin{array}{c}
\sin \theta_{s} \cos \phi \\
\sin \theta_{s} \sin \phi \\
\cos \theta_{s}
\end{array}\right] .
\end{aligned}
$$

The direction of the photon packet $\mathbf{u}_{i}$ together with its free path length $l_{i}$ determines the point at which the next scattering event takes place: $\mathbf{r}_{i 1}=\mathbf{r}_{i}+l_{i} \mathbf{u}_{i}$. At this point a new direction and path length are determined, and the steps described above are repeated until the photon exits the scattering medium.

The polar scattering angle is sampled from a scattering phase function ${ }^{24}$ :

$$
p\left(\mathbf{k}_{i}, \mathbf{k}_{s}\right)=\frac{\sigma\left(\mathbf{k}_{i}, \mathbf{k}_{s}\right)}{\int_{4 \pi} \sigma\left(\mathbf{k}_{i}, \mathbf{k}_{s}\right) \mathrm{d} \Omega_{s}} .
$$

Depending on the purpose of the study, the Mie,1,2,43 Rayleigh-Gans, ${ }^{1,2}$ or Henyey-Greenstein ${ }^{44}$ phase function is typically used. The change in polar angle $\theta_{s}$ is determined from its inverse cumulative probability density function (iCPDF), ${ }^{45} \theta_{s}=\mathrm{CPDF}^{-1}(\xi)$. When the analytical form of the iCPDF is not available, CPDF is stored in a look-up table 45,46 and the inverse transformation is performed at each scattering event.

The first step is to validate the $\mathrm{MC}$ technique against analytical results. To do this we consider isotropic scattering. It is possible to obtain the corresponding analytical results when the scattering is isotropic. Also, the number of parameters to be considered is reduced. In this case $\operatorname{CPDF}\left(\theta_{s}\right)$ is obtained from $\cos \left(\theta_{s}\right)=(2 \xi-1)$, and $\operatorname{CPDF}(\phi)$ is obtained from $\phi=2 \pi \xi$.

In the second step, $\mathrm{MC}$ simulations were performed with two different anisotropic phase functions, based, respectively, on spherical droplets of 1 and $15 \mu \mathrm{m}$ in diameter. These phase functions were deduced from Mie-scattering theory, considering a realistic spray diagnostic problem. The source wavelength $\lambda$ is $532 \mathrm{~nm}$, the refractive index of the droplet is 1.4 
$+0.0 i$, and the refractive index of the surrounding medium is $1+0.0 i$. The corresponding factor of anisotropy $g$ equals 0.782 for the $1-\mu \mathrm{m}$ sphere and 0.818 for the $15-\mu \mathrm{m}$ sphere. This represents a spray of hydrocarbon fuel in air.

Photon packets are assumed to have been detected if they reach the detector $D$ with an incident angle $\theta_{s}$ less than detector acceptance angle $\theta_{a}\left(\theta_{s} \leq \theta_{a}\right)$. For each detected photon packet the scattering order and the distance $h$ from the incident beam are stored in the data file. The intensity of different scattering orders is then determined by the number of photons recorded and can be plotted as a function of $h$.

If the detector acceptance angle is very small $\left(\theta_{a}\right.$ $<2^{\circ}$ ), the number of photon packets detected is low and the data show strong statistical fluctuations. To obtain less noisy results for the small acceptance angle $\left(\theta_{a} \sim 0^{\circ}\right)$, we use a semianalytical MC scheme. ${ }^{20,47}$ This scheme uses the probability $W$ that the scattered photon packet strikes the detector at normal incidence:

$$
W=p\left(\mathbf{k}_{d}-\mathbf{k}^{\prime}\right) \mathrm{d} \Omega_{d} \exp \left(-\mu_{t}, d\right) .
$$

Here $\mathbf{k}_{d}$ is the vector of normal toward the detector, $\mathrm{d} \Omega_{d}$ is the elementary solid angle spanning a line normal to the detector, and $d$ is the distance between the photon scattering and the detector. $p\left(\mathbf{k}_{d}-\mathbf{k}^{\prime}\right)$ is the scattering phase function, constant for isotropic scattering: $p\left(\mathbf{k}_{d}-\mathbf{k}^{\prime}\right)=1 / 4 \pi$. The intensity of the scattering orders is then obtained by calculation of this probability for all the scattering events. This significantly reduces the computational time required for a given noise level (by a factor of $\sim 100$ ) and allows accurate comparisons between the $\mathrm{MC}$ and the analytical approaches.

The MC computational time is directly dependant on the optical parameters of the scattering medium, on the geometry of this medium, and on the number of photons sent. Considering anisotropic scattering process with $\mu_{s}=0.08 \mathrm{~mm}^{-1}$, a typical MC computational time of $\sim 8$ is required for an amount of 1 billion of photons sent with a P4 2.5-GHz CPU.

\section{Results and Discussion}

The results of the MC simulation and the analytical calculation of $I_{(2)}(h)$ are presented in Figs. 3(a) and $3(\mathrm{~b})$. The circles represent the results of the MC simulation, the dashed curve shows the results of an approximate analytical calculation, and the solid curve is the exact analytical calculation. To calculate the intensity of double scattering by Eq. (7), we introduce the cylindrical coordinate frames $\left(l_{1}, r_{1}, \phi_{1}\right)$ and $\left(l_{2}, r_{2}, \phi_{2}\right)$, i.e.,

$$
\begin{aligned}
& \mathbf{r}_{1}=\left(r_{1} \cos \phi_{1}, r_{1} \sin \phi_{1}, l_{1}\right), \\
& \mathbf{r}_{2}=\left(l_{2}, h+r_{2} \cos \phi_{2}, r_{2}, \sin \phi_{2}\right) .
\end{aligned}
$$

If $h \gg R_{1}$ and $R_{2}$, and the source-detector intersect angle $\beta=90^{\circ}$, Eq. (7) is significantly simplified and
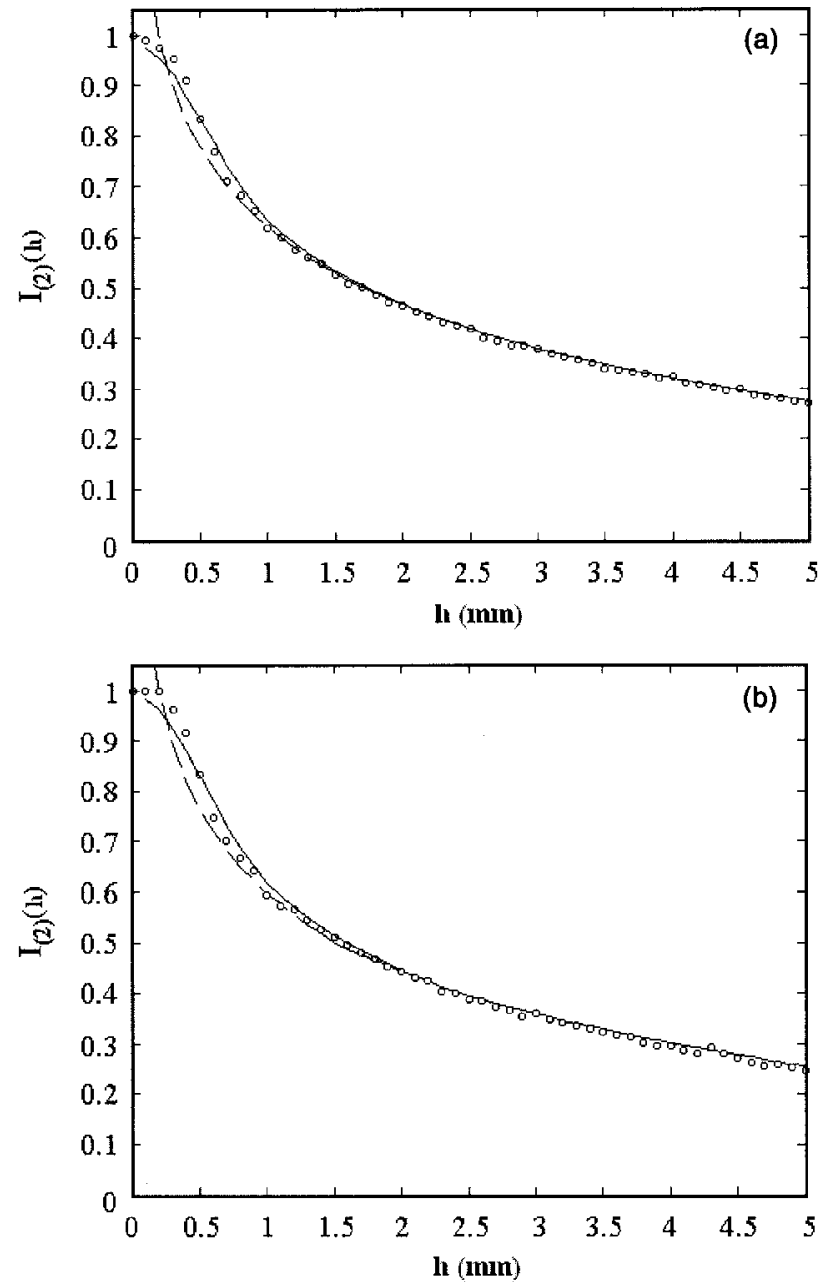

Fig. 3. Intensity of the double light scattering $I_{(2)}$ with respect to the distance $h$ between $V_{1}$ and $V_{2}$ : (a) $\mu_{s}=0.04 \mathrm{~mm}^{-1}$, (b) $\mu_{s}$ $=0.16 \mathrm{~mm}^{-1}$. The solid curve represents the results of calculation by the exact Eq. (7), the dotted curve by the approximate Eq. (13), and $(O)$ are the results of the MC simulation.

can be written as

$$
\begin{aligned}
I_{(2)}(h)= & A \pi^{2} R_{1}{ }^{2} R_{2}{ }^{2} \int_{-L_{1 / 2}}^{L_{1 / 2}} \mathrm{~d} x \int_{-L_{2 / 2}}^{L_{2 / 2}} \mathrm{~d} z \frac{1}{x^{2}+z^{2}+h^{2}} \\
& \times \exp \left\{-\mu_{t}\left[L+z-x+\left(x^{2}+z^{2}+h^{2}\right)\right]^{1 / 2}\right\} .
\end{aligned}
$$

It can be seen that, for the low scattering ( $\mu_{s}$ $=0.04 \mathrm{~mm}^{-1}$ ) of the medium, the results of the MC and analytical calculations agree reasonably well [Fig. 3(a)]. Similar agreement is obtained as well for higher scattering of the medium $\left(\mu_{s}=0.16 \mathrm{~mm}^{-1}\right)$, especially for $h>2 \mathrm{~mm}$ [see Fig. 3(b)]. The agreement is not total as the analytical results neglect scattering orders higher than 2, whereas the MC results include these. The dashed curves show the results of the analytical calculations with the approximate form of $I_{(2)}$ [Eq. (13)]. Equation (13) reduces to $I_{(2)}(h) \sim|\ln (h)|$ for $h \rightarrow 0$, i.e., it predicts that the intensity of scattering goes to infinity, which 


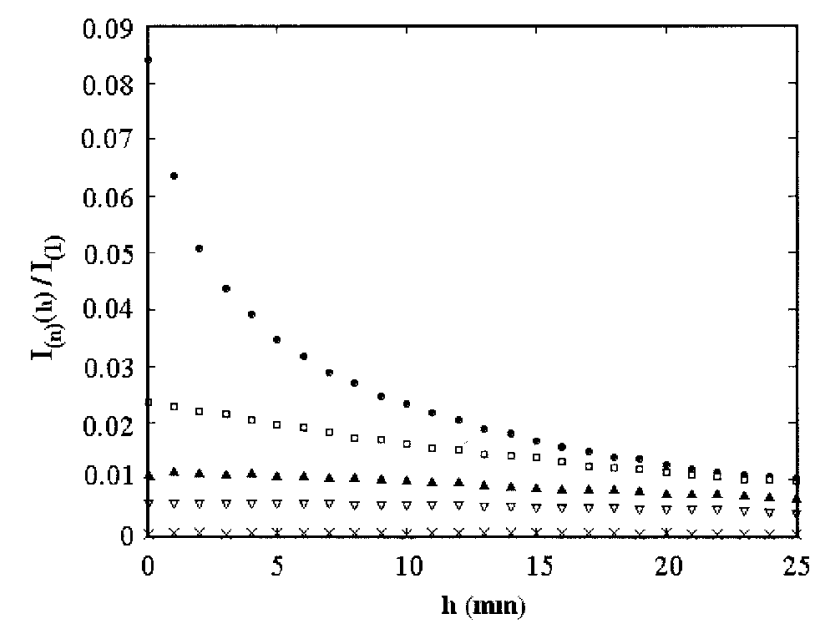

Fig. 4. Intensity of different scattering orders $I_{(n)}$ as a function of $h$ :, double scattering $I_{(2)}$; $\square$, triple scattering $I_{(3)}$, $\mathbf{\text { , fourth-order }}$ scattering $I_{(4)} ; \nabla$, fifth-order scattering $I_{(5)}$, and x, tenth-order scattering $I_{(10)}$. The intensity of each scattering order is normalized to the intensity of single scattering $I_{(1)}$ at $h=0$.

is unphysical. The discrepancy between the exact Eq. (7) [solid curves in Figs. 3(a) and 3(b)] and the approximate Eq. (13) begins at $h=1.2 \mathrm{~mm}$, which is close to the diameter of the cylinders.

Analytical expressions for the high scattering orders (i.e., $I_{(3)}, I_{(4)}$, and so on) differ from the expressions for $I_{(1)}, I_{(2)}$ [see Eqs. (1) and (2)] and involve multiorder integrals. This is due to presence of the intermediate integration over the total scattering volume. However, with the MC technique it is possible to evaluate these higher scattering orders. The third, fourth, fifth, and tenth scattering orders calculated by the MC technique for the low scattering medium $\left(\mu_{s}=0.04 \mathrm{~mm}^{-1}\right)$ are presented in Fig. $4 . I_{(2)}(h)$ is also included for comparison. All the calculated intensities are normalized to the value of the single lightscattering intensity $I_{(1)}$ at $h=0 . I_{(1)}$ dominates the other scattering orders. It should be pointed out here that the high-order integrals do not possess a logarithmic singularity as predicted by Eq. (13) and return smooth functions for small $h .48$ This is clearly demonstrated by the results of the MC simulation, i.e., $I_{(n)}, n \geq 3$ are smooth functions of $h$ (see Fig. 5). The intensity of the higher scattering orders falls with increasing $h: I_{(3)}$ falls by a factor of 2.5 when $h$ varies from 0 to $25 \mathrm{~mm}, I_{(4)}$ decreases by $1.4, I_{(5)}$ decreases by 1.1 , and $I_{(10)}$ is effectively equal to zero. For comparison, $I_{(2)}$ is reduced by a factor of 9 in the same interval.

Figure 5 shows the effect of the detector acceptance angle $\theta_{a}$ on the detected intensities of different scattering orders. These simulations are based on a source-detector angle $\beta$ equal to $90^{\circ}$. Single and double scattering are strongly dependent on acceptance angle [see Figs. 5(a) and 5(b)]. However, higher scattering orders (e.g., third and fifth) are much less sensitive to $\theta_{a}$ [see Figs. 5(c) and 5(d)]. This can be explained by a consideration of the localization of the trajectories that the photon packets take traveling between the source and the detector. For single and double scattering, to enter a detector with a small acceptance angle these trajectories are clustered into a compact locus. The locus of trajectories with high orders of scattering will be less compact.

The influence of the scattering coefficient on the detection of different scattering orders $n$ (where $n$ $=\{3-5,10\})$ is illustrated in Fig. 6 . The ratio of the intensity of the $n$th order of scattering to that of single scattering at $h=0$, i.e., $I_{(n)}(0) / I_{(1)}(0)$, is plotted. This ratio increases monotonically with scattering coefficient $\mu_{s}$. With $\mu_{s}<0.14 \mathrm{~mm}^{-1}$, the intensity of the scattering order falls off as the scattering order increases from 3 to 10 . In the interval $\mu_{s}$ $\sim 0.14-0.15 \mathrm{~mm}^{-1}$ the intensities of orders $3-10$ become very similar, and for higher $\mu_{s}$ an inversion of the intensities of the scattering orders takes place (see Fig. 6). It is clear that the contribution of high scattering orders is significant $(\approx 15-20 \%$ of the single-scattering intensity at $h=0$ ) when $\mu_{s}$ $\sim 0.13-0.15 \mathrm{~mm}^{-1}$. This illustrates the transition from the single-scattering regime to the multiplescattering regime, where the light transport can be described by the diffusion approximation. The results obtained agree well with the experimental results. ${ }^{49}$ It is deduced from Fig. 6 that an isotropic scattering system with the geometry described here enters the intermediate scattering regime when $\mu_{s} \sim 0.16 \mathrm{~mm}^{-1}$.

$I_{(2)}(h)$ is plotted in Fig. 7 for isotropic scattering with $\mu_{s}=0.04$ and $0.15 \mathrm{~mm}^{-1}$ as well as for anisotropic scattering with $\mu_{s}=0.04 \mathrm{~mm}^{-1}$. For each curve, $I_{2}(h)$ is normalized by the value at $h=0$. The curves corresponding to isotropic scattering have an acceptance angle of effectively zero. For the anisotropic scattering (with an acceptance angle of $2^{\circ}$ ) the decrease of intensity $I_{(2)}$ with $h$ is stronger, even for a low scattering coefficient.

The fraction of the total intensity contributed by different scattering orders $I_{(n)} / I_{(\text {tot })}$ is plotted as a function of detector acceptance angle in Fig. 8 for $h$ $=0$ and $\mu_{s}=0.04 \mathrm{~mm}^{-1}$. For isotropic scattering [Fig. 8(a)], single scattering dominates at small $\theta_{a}\left(\theta_{a}\right.$ $<20^{\circ}$ ). The contribution of single and multiple (double plus higher orders) scattering becomes equal at a detector acceptance angle $\theta_{a} \approx 15^{\circ}$. As expected, the contribution of single scattering decreases with increasing detector aperture; and for $\theta_{a}>15^{\circ}$, multiple scattering dominates the detected signal. This is logical, as with increasing $\theta_{a}$ the detector collects light scattered from an increasing volume of the medium outside the cylindrical source beam.

In the case of anisotropic scattering the singlescattering intensity reaches only a maximum of $27 \%$ and the double light-scattering intensity dominates the detected signal [Fig. 8(b)]. This clearly demonstrates the effect of the phase function on the detection geometry. The diameter of particles considered here is relatively large $(a=15 \mu \mathrm{m})$, and the Mie phase function shows strong forward scattering. The probability for a photon to keep virtually the same 

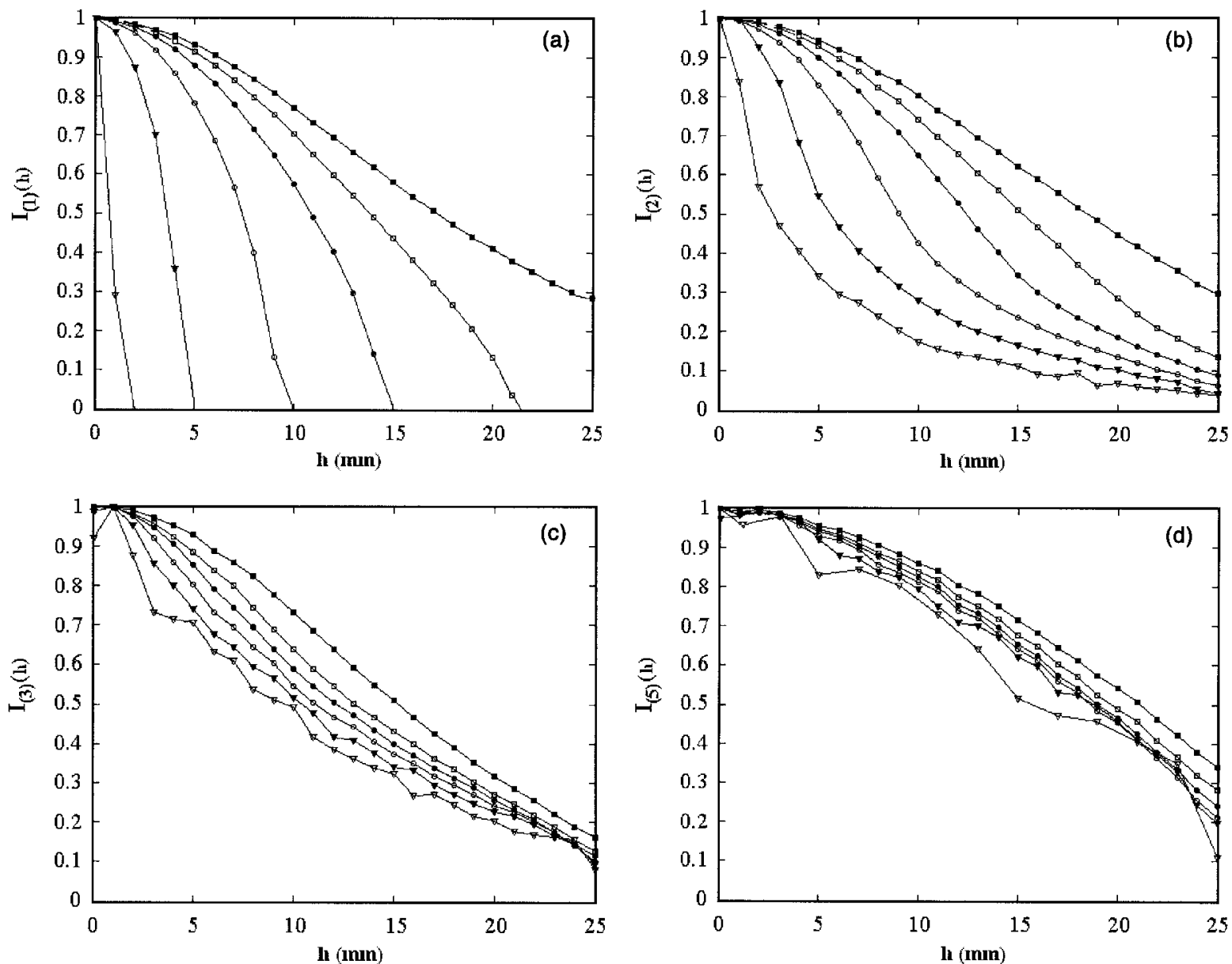

Fig. 5. Normalized intensity of different scattering orders versus $h$ : (a) single light scattering, (b) double scattering, (c) triple scattering, (d) fifth-order scattering. The symbols represent different values of the detector numerical aperture: $\nabla, 2^{\circ} ; \mathbf{v}, 10^{\circ} ; \bigcirc, 20^{\circ} ; \mathbf{0}, 40^{\circ} ; \square, 60^{\circ}$; 口, $90^{\circ}$.

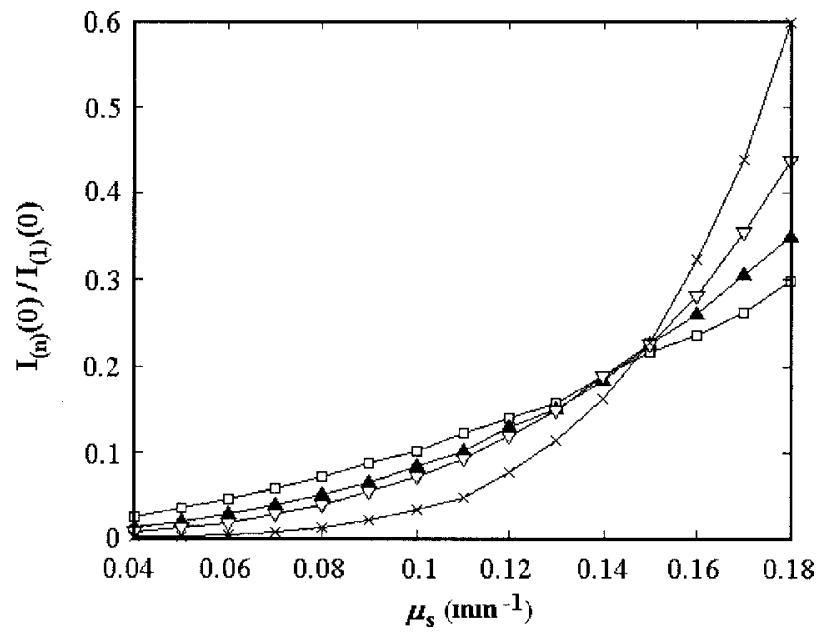

Fig. 6. Normalized intensities of $n$th scattering orders and singlescattering intensity at $h=0, I_{(n)}(0) / I_{(1)}(0)$ as a function of scattering coefficient $\mu_{s}: \square$, triple scattering $I_{(3)} ; \mathbf{\wedge}$, fourth-order scattering $I_{(4)} ; \nabla$, fifth-order scattering $I_{(5)}$; x, tenth-order scattering $I_{(10)}$.

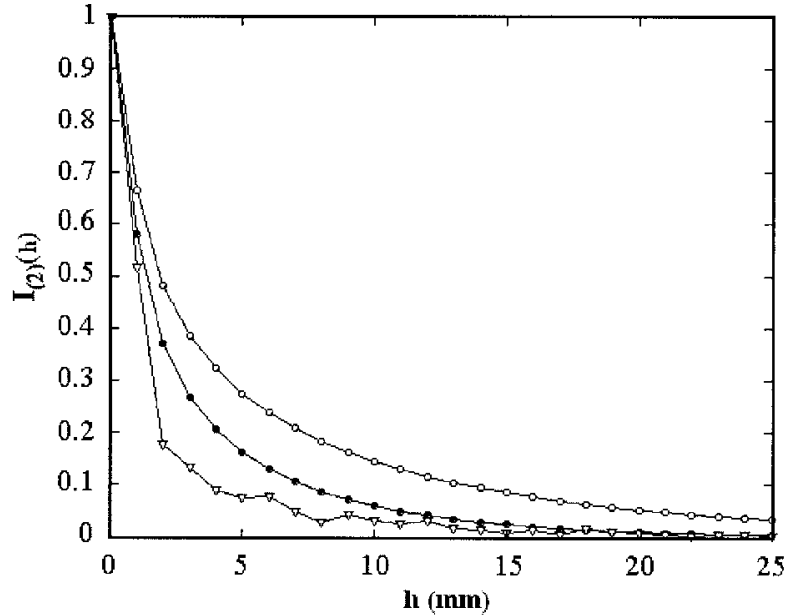

Fig. 7. Normalized intensity of the double light scattering $I_{(2)}$ with respect to the distance $\mathrm{h}$ between $V_{1}$ and $V_{2}: \bigcirc$, isotropic scattering with $\mu_{s}=0.04 \mathrm{~mm}^{-1} ; \boldsymbol{\bullet}$, isotropic scattering with $\mu_{s}$ $=0.16 \mathrm{~mm}^{-1} ; \nabla$, anisotropic scattering with $\mu_{s}=0.04 \mathrm{~mm}^{-1}$ for particle diameter $a=15 \mu \mathrm{m}$. 

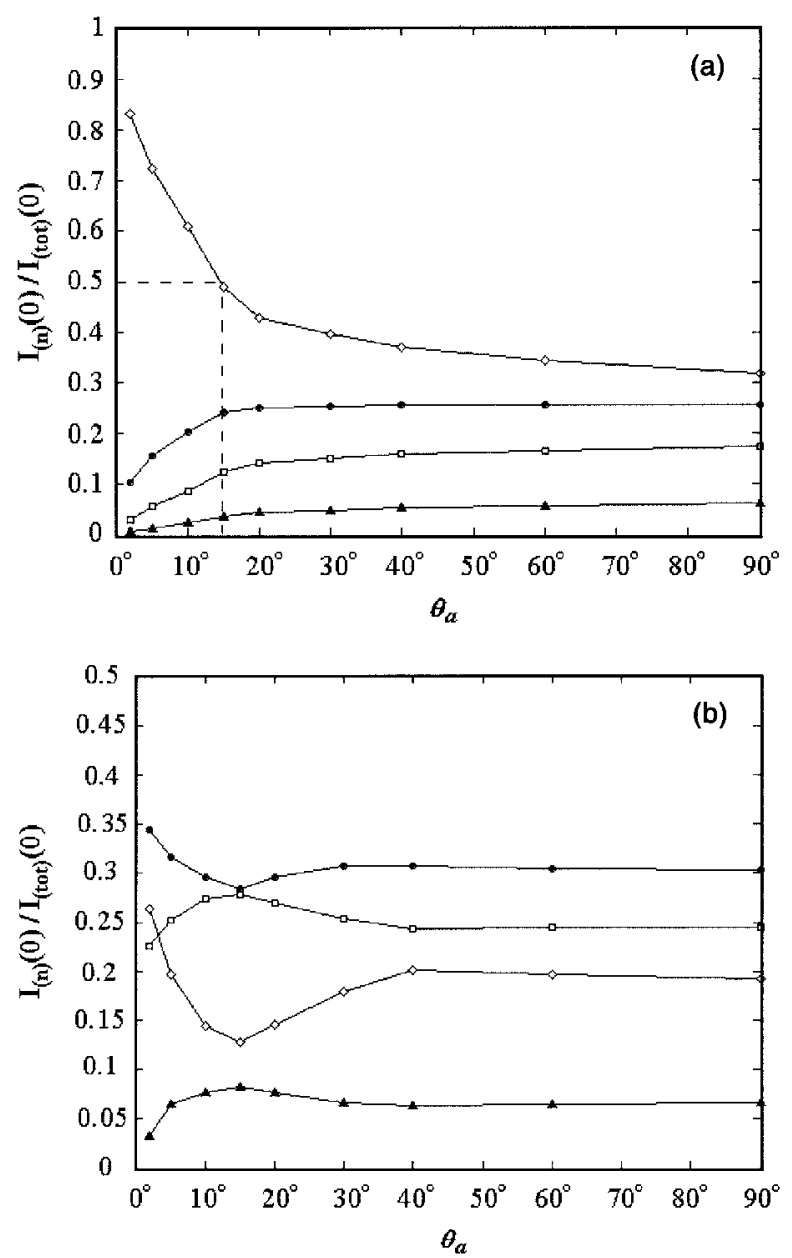

Fig. 8. Results of MC calculation of the different scattering order intensities $I_{(n)} / I_{\text {(tot) }}$ at $h=0$ versus the acceptance angle $\theta_{a}$ : (a) isotropic scattering with $\mu_{s}=0.04 \mathrm{~mm}^{-1}$, (b) anisotropic scattering with $\mu_{s}=0.04 \mathrm{~mm}^{-1}$ for particle diameter $a=15 \mu \mathrm{m}$. The symbols represent different scattering orders: $\diamond$, single scattering $I_{(1)} ; \bullet$ double scattering $I_{(2)} ; \square$, triple scattering $I_{(3)}, \mathbf{\Delta}$, fourth-order scattering $I_{(4)}$.

direction after a scattering event is high. If a narrow acceptance angle is considered, this means that the amount of multiple scattering detected will mainly occur in the volumes $V_{1}$ or $V_{2}$ (Fig. 2) and single scattering detected will take place at the intersection of $V_{1}$ with $V_{2}$. If a photon is initially scattered in the direction of the detector, it is unlikely that any other scattering events occurring between this first scatter and the detector will deviate the photon's trajectory enough to prevent it being detected because of the high probability of forward scattering. The intensity of double and triple scattering detected thus increases [Fig. 8(b)]. The influence of $\theta_{\alpha}$ is then not as important as for isotropic scattering and is not as useful for the filtering of the signal to extract single scattering.

The effect of the source-detector intersection angle $\beta$ on the detection of single scattering is investigated Fig. 9. The results are presented for isotropic scattering and two different anisotropic phase functions cor-
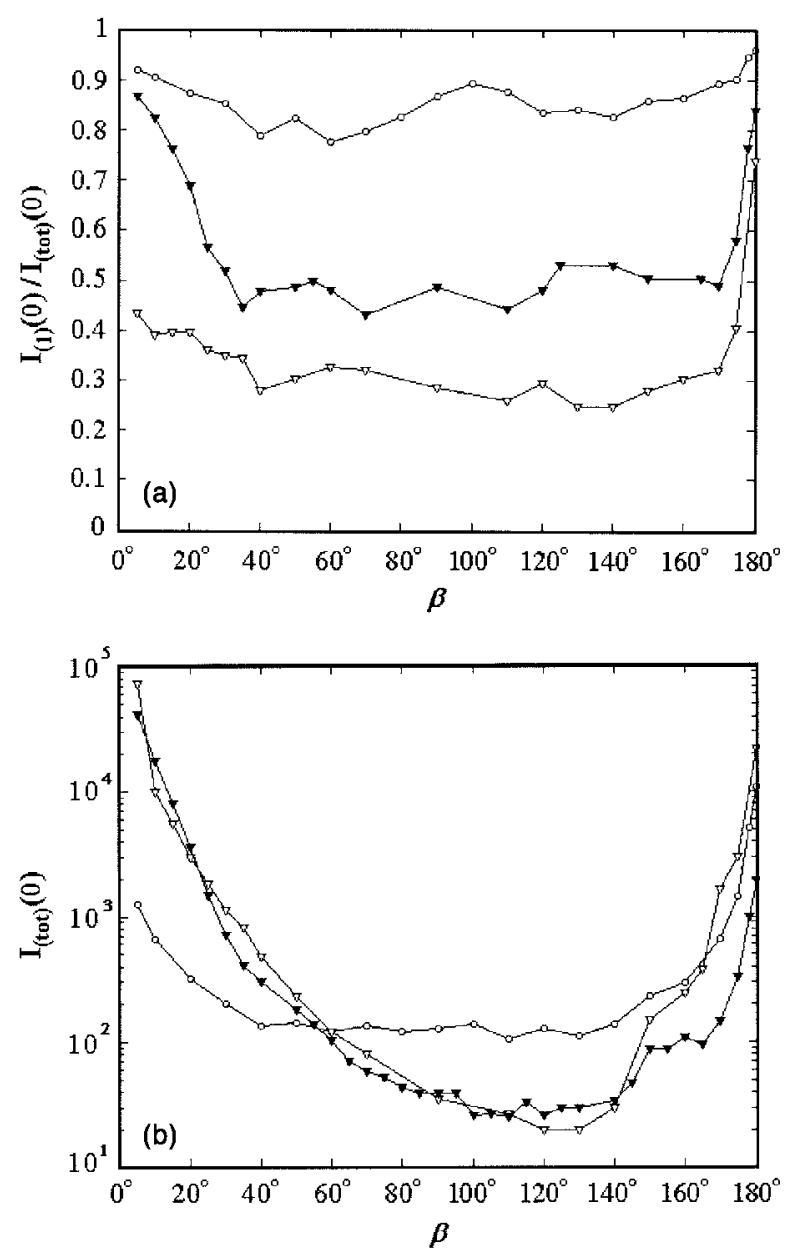

Fig. 9. Effect of the source-detector angle $\beta$ on the singlescattering detection at $h=0$ with an acceptance angle of $2^{\circ}: \bigcirc$, isotropic scattering with $\mu_{s}=0.04 \mathrm{~mm}^{-1} ; \nabla$, anisotropic scattering with $\mu_{s}=0.04 \mathrm{~mm}^{-1}$ for particle diameter $a=15 \mu \mathrm{m}$; $\nabla$, anisotropic scattering with $\mu_{s}=0.04 \mathrm{~mm}^{-1}$ for particle diameter $a$ $=1 \mu \mathrm{m}$. (a) Amount of single scattering $I_{(1)} / I_{\text {(tot) }}$ detected versus $\beta$. (b) Total intensity detected versus $\beta$.

responding to a 1- and $15-\mu \mathrm{m}$ diameter of particles. The acceptance angle $\theta_{\alpha}$ is $2^{\circ}$ and $\mu_{s}$ is $0.04 \mathrm{~mm}^{-1}$. The fraction of the total intensity contributed by single scattering $I_{(1)} / I_{\text {(tot) }}$ as a function of $\beta$ is plotted in Fig. 9(a). It can be seen that for isotropic scattering the fraction of single scattering in the total detected signal is insensitive to $\beta$ and remains between $80 \%$ and $95 \%$. On the contrary, the effect of $\beta$ on the detection of single scattering is significant for anisotropic scattering. It can be seen that forwardscattering $\left(\beta\right.$ close to $\left.0^{\circ}\right)$ and backscattering detection ( $\beta$ close to $180^{\circ}$ ) are most efficient for the extraction of single scattering [Fig. 9(a)]. For particles of $15 \mu \mathrm{m}$ in diameter the single-scattering intensity detected reaches $44 \%$ for $\beta=5^{\circ}$ and $76 \%$ for $\beta=180^{\circ}$.

The detection of single scattering with forward detection $\left(\beta=0^{\circ}\right)$ is more efficient for small particles (88\% of $I_{1}$ with $a=1 \mu \mathrm{m}$ ) than for large particles (44\% of $I_{1}$ with $a=1 \mu \mathrm{m}$ ). With $\beta=90^{\circ}$, singlescattering intensity reaches only $\sim 30 \%$ for $a$ 


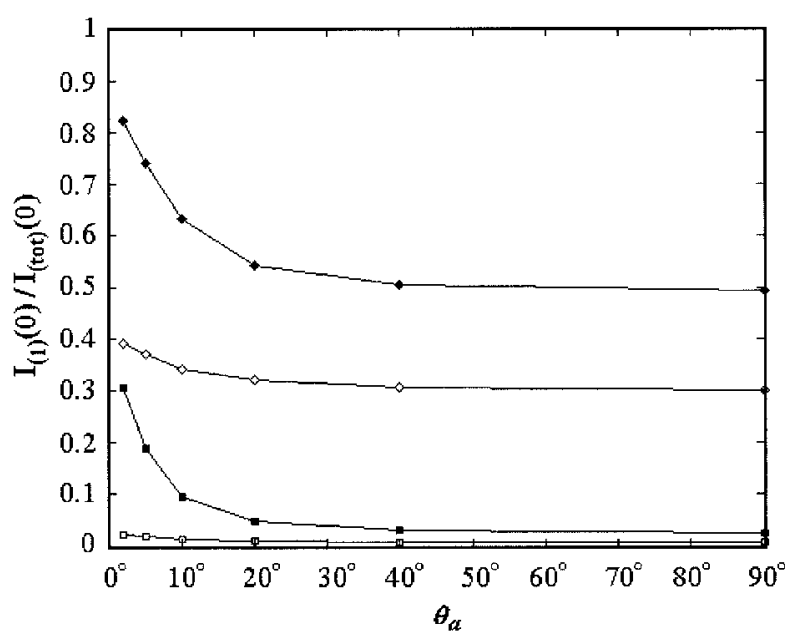

Fig. 10. Results of $I_{(1)} / I_{(\mathrm{tot})}$ versus acceptance angle $\theta_{a}$ for anisotropic scattering with $\beta=10^{\circ}$ and with different scattering coefficients: $\diamond, \mu_{s}=0.04 \mathrm{~mm}^{-1}$ for particle diameter $a=15 \mu \mathrm{m}$; $\square$, $\mu_{s}=0.16 \mathrm{~mm}^{-1}$ for particle diameter $a=15 \mu \mathrm{m}$; $(\square), \mu_{s}$ $=0.04 \mathrm{~mm}^{-1}$ for particle diameter $a=1 \mu \mathrm{m} ; \boldsymbol{\square}, \mu_{s}=0.16 \mathrm{~mm}^{-1}$ for particle diameter $a=1 \mu \mathrm{m}$.

$=15 \mu \mathrm{m}, \sim 50 \%$ for $a=1 \mu \mathrm{m}$, but $\sim 85 \%$ for isotropic scattering. Perpendicular detection of first scattering orders seems then more appropriate for scattering processes close to isotropic scattering. However, the backscattering detection $\left(\beta=180^{\circ}\right)$ allows a high amount of single scattering to be detected for isotropic and for both anisotropic cases: $84 \%$ of $I_{1}$ with $a$ $=1 \mu \mathrm{m}$ and $74 \%$ of $I_{1}$ with $a=15 \mu \mathrm{m}$.

Figure 9(b) shows the total intensity detected as a function of the source-detector angle $\beta$ for $\mu_{s}$ $=0.04 \mathrm{~mm}^{-1}$. This total intensity reaches a maximum for the forward detection with both of the anisotropic scattering processes considered. However, $I_{\text {(tot) }}$ is strong for isotropic scattering at $\beta=90^{\circ}$ as well as at forward scatter and backscatter. Backscattering intensity is strong for all three scattering processes investigated.

Considering Figs. 9(a) and 9(b) together it seems that forward-scattering detection allows detection of both a high fraction of single scattering and a strong total signal. It is possible to detect a signal that is strongly dominated by single scattering at nearforward and near-backward scatter. If high spatial resolution is desired, care must be taken that $\beta$ (in the case of forward scatter) or $\beta$ close to $180^{\circ}$ (in the case of backscatter) is greater than the acceptance angle so that the volume in which the detected signal is scattered is kept small. In the case of forward scattering, keeping $\beta$ greater than the acceptance angle avoids the detection of unscattered light.

In Fig. 10 we show the fraction contributed by single scattering for a low $\left(\mu_{s}=0.04 \mathrm{~mm}^{-1}\right)$ and high $\left(\mu_{s}=0.16 \mathrm{~mm}^{-1}\right)$ scattering medium with anisotropic scattering and a source-detector angle equal to $10^{\circ}$. It can be seen that, if the particles are large, the influence of the acceptance angle is not so strong. For $\mu_{s}=0.16 \mathrm{~mm}^{-1}$, detection of single scattering reaches a maximum of $30 \%$ for particles of $1 \mu \mathrm{m}$ in diameter, against only $\sim 2 \%$ for particles of $15 \mu \mathrm{m}$.

\section{Summary and Conclusion}

The analysis of different scattering orders in the intermediate scattering regime has been carried out for a randomly inhomogeneous scattering medium with a novel crossed source-detector geometry. The results demonstrate good agreement between the analytical and MC techniques. The influence of detector numerical aperture on the intensity of the different scattering orders is shown quantitatively. Particular attention is paid to the difficult intermediate regime, where the scattering power of the medium is too high for single scattering to be assumed but is too low for the diffusion approximation to be applied. The results show the characteristics of the transition from single to multiple scattering. The agreement between analytical and MC results validates use of the MC approach in the intermediate scattering regime. The method can be applied to verify analytical results against experimental results indirectly through $\mathrm{MC}$ calculations that account for imperfections of the experiment that are difficult to represent analytically.

The geometry chosen for the optical experiment is interesting in that it allows the experimenter to measure the intensity of single and double light scattering separately. It is then possible to extract information on the absolute values of the medium optical properties from the ratio of single-to-double scattering intensity. If the double and single lightscattering intensity are of the same order of magnitude, then the higher-order scattering, $I_{(n), n \geq 3}$, must be taken into account. Both analytical and MC calculations show a strong logarithmic dependence of double light scattering from the distance $h$ between the illuminating and the collecting volumes. Dependence of higher scattering orders is weaker and can be considered constant compared with the single order. The total intensity of the scattered light $\left.I_{(\mathrm{tot})}\right)(h)$ can then be considered as

$$
I_{(\mathrm{tot})}(h)=I_{(1)}\left(r_{0}\right)+I_{(2)}(h)+I_{(p)}(h),
$$

where $r_{0} \in V_{\text {single }}$, where $V_{\text {single }}$ is an effective volume of single light scattering, and $I_{p}(h)=\sum_{n \geq 3} I_{(n)}$.

The separation between single and higher orders of scattering is not perfect, but the MC simulations allow us to develop an iterative procedure to determine the contributions of each scattering order to the total intensity separately, thus completing the task of experimental data processing. In this procedure the optimum detector acceptance angle and sourcedetector separation $h$ would be estimated from MC results similar to those presented here. Then the experiment will be performed in these optimum conditions, and properties of the medium (particle size and concentration) will be estimated from experimental data assuming that third- and higher-order scattering is negligible. With these estimates used as input data for a second MC simulation, the user would be able to estimate the intensity of the third- and higher- 
order scattering parameters, which can be used to determine an improved estimate of the particle properties. The procedure would be iterated until the estimated properties converged.

The MC method was successfully applied to calculate the intensities of different orders of scattering. These results can be used to infer the optical parameters of inhomogeneous media with the experimental geometry shown in Fig. 2. The proposed experimental geometry can be used in a practical experiment to measure the particulate scattering field properties. The illumination and detection volumes cannot be ideally cylindrical; but with a laser with a high confocal parameter and a tightly collimated detector they can be near cylindrical, and the MC code can be used to quantify the effect of these experimental imperfections.

Another advantage of the suggested approach is that both the dependence of the scattered light intensity on the distance parameter $h$ and the angular intensity dependence, i.e., the dependence on $\theta_{a}$, can be obtained. In addition, the results validate and support use of the MC method in the intermediate scattering regime and provide details of transition from low scattering (where the intensity of a scattering order drops as the order increases) to multiple scattering (where the intensity increases with order).

We have demonstrated the agreement between MC and analytical results in a system of isotropic scatterers. We then considered anisotropic scatter and deduced the experimental parameters (detector acceptance angle, source-detector angle and offset) that deliver the optimum discrimination between the first and higher scattering orders.

The MC procedure can be readily generalized to cover spatially inhomogeneous media and systems containing particles of different sizes (polydisperse media). As a next step in the current study, we intend to include the polarization in the MC code verified here, apply the code to the estimation and suppression of errors in existing spray diagnostics, and develop new optical spray diagnostic techniques.

\section{Appendix A: Nomenclature}

\section{Symbols}

$a$, particle diameter;

$c$, speed of light in vacuum;

$h$, distance between the hypothetical volumes of incident and scattered light;

$G(\mathbf{q})$, correlation function of the permittivity fluctuations;

$h$, distance between the hypothetical volumes of incident and scattered light;

$\mathbf{k}_{s}$ and $\mathbf{k}_{i}$, wave vectors of the incident and scattered light;

$\mathbf{k}^{\prime}$, intermediate wave vector;

$\mathbf{k}_{d}$, vector of normal toward the detector;

$I_{(n)}$, intensity of the $n$th scattering order;

$I_{(\mathrm{tot})}$, total intensity of the scattered light;

$l_{i}$, path length of a photon packet; $l_{1}$ and $l_{2}$, paths before and after the scattering event;

$L_{1}, L_{2}$, lengths of $V_{1}$ and $V_{2}$ cylinders;

$N$, particle density number;

$p\left(\mathbf{k}_{i}, \mathbf{k}_{s}\right)$, phase function;

$g$, factor of anisotropy;

q, scattering wave vector;

$R_{1}, R_{2}$, radii of $V_{1}$ and $V_{2}$ cylinders;

$\mathbf{u}_{i}$ and $\mathbf{u}_{s}$, unit vectors;

$V_{1}$ and $V_{2}$, cylindrical volumes of incident and scattered laser beams.

\section{Greek Symbols}

$\beta$, source-detector angle;

$\Delta \varepsilon$, difference of permittivities of particles and host medium;

$\theta_{a}$, detector acceptance angle;

$\theta_{s}$, polar angle;

$\varphi$, azimuth angle;

$\lambda$, wavelength of the light source;

$\mu_{t}$, extinction coefficient;

$\mu_{s}$ and $\mu_{a}$, scattering and absorption coefficients;

$\omega$, cycle frequency;

$\xi$, random number uniformly distributed between 0 and 1 ;

$\mathrm{d} \Omega_{d}$, the elementary solid angle.

\section{Subscripts}

$\alpha$ and $\beta$, indices of polarization of the incident and scattered light;

$i$, sequential number of scattering events.

We are grateful to V. L. Kuzmin for valuable comments and discussions. This study was partially supported by the Royal Society (project 15298), NATO (project PST.CLG.979652), the Engineering and Physical Sciences Research Council (UK) (grant GR/R92653), and an Overseas Research Scholarship.

\section{References}

1. H. C. van de Hulst, Light Scattering by Small Particles (Dover, New York, 1981).

2. C. Bohren and D. Huffman, Absorption and Scattering of Light by Small Particles (Wiley, New York, 1983).

3. A. Ishimaru, Wave Propagation and Scattering in Random Media (Oxford U. Press, Oxford, UK, 1997).

4. B. A. van Tiggelen and S. E. Skipetrov, Wave Scattering in Complex Media: From Theory to Applications, Vol. 107 of NATO Science Series: II: Mathematics, Physics and Chemistry (Kluwer Academic, Dordrecht, The Netherlands, 2003).

5. V. L. Kuz'min and V. P. Romanov, "Coherent phenomena in light scattering from disordered systems," Usp. Fiz. Nauk 39, 231-260 (1996).

6. J. Q. Shen and U. Riebel, "Extinction by a large spherical particle located in a narrow Gaussian beam," Part. Part. Syst. Charact. 18, 254-261 (2001).

7. G. Gouesbet, B. Maheu, and G. Grehan, "Light scattering from a sphere arbitrarily located in a Gaussian beam, using a Bromwich formulation," J. Opt. Soc. Am. A 5, 1427-1443 (1988).

8. Z. Ma, H. G. Merkus, H. G. van der Veen, M. Wong, and B. Scarlett, "On-line measurement of particle size and shape using laser diffraction," Part. Part. Syst. Charact. 18, 243-247 (2001).

9. M. Kocifaj and M. Drzik, "Retrieving the size distribution of 
microparticles by scanning the diffraction halo with a mobile ring-gap detector," J. Aerosol. Sci. 28, 797-804 (1997).

10. M. Kerker and D. D. Cooke, "Remote sensing of particle size and refractive index by varying the wavelength," Appl. Opt. 15, 2105-2111 (1976).

11. A. R. Jones, "Scattering of electromagnetic radiation in particulate laden fluids,” Prog. Energy Combust. Sci. 5, 73-96 (1979).

12. W. C. Hinds, Aerosol Technology: Properties, Behavior, and Measurement of Airborne Particles (Wiley, New York, 1982).

13. F. Zhao, Z. Gong, H. Hu, M. Tanaka, and T. Hayasaka, "Simultaneous determination of the aerosol complex index of refraction and size distribution from scattering measurements of polarized light," Appl. Opt. 36, 7992-8001 (1997).

14. M. C. Jermy and D. A. Greenhalgh, "Planar dropsizing by elastic and fluorescence scattering in sprays too dense for phase Doppler measurement," Appl. Phys. B 71, 703-710 (2000).

15. I. M. Sobol', The Monte Carlo Method (University of Chicago, Chicago, Ill., 1974).

16. G. I. Marchuk, G. A. Mikhailov, M. A. Nazaraliev, R. A. Darbinjan, B. A. Kargin, and B. S. Elepov, The Monte Carlo Method in Atmospheric Optics (Springer, Berlin, 1980).

17. V. P. Kandidov, "Monte Carlo methods in nonlinear statistical optics," Usp. Fiz. Nauk 39, 1243-1272 (1996).

18. S. A. Prahl, M. Keijzer, S. L. Jacques, and A. J. Welch, "A Monte Carlo model of light propagation in tissue," in Dosimetry of Laser Radiation in Medicine and Biology, G. J. Müller and D. H. Sliney, eds., Vol. IS5 of the SPIE Institute Series (SPIE, Bellingham, Wash., 1989), pp. 102-111.

19. I. V. Meglinsky and S. J. Matcher, "Modeling the sampling volume for skin blood oxygenation measurements," Med. Biol. Eng. Comput. 39, 44-50 (2001).

20. R. R. Meier, J.-S. Lee, and D. E. Anderson, "Atmospheric scattering of middle UV radiation from an internal source," Appl. Opt. 17, 3216-3225 (1978).

21. C. Lavigne, A. Robin, V. Outters, S. Langlois, T. Girasole, and C. Roze, "Comparison of iterative and Monte Carlo methods for calculation of the aureole about a point source in the Earth's atmosphere," Appl. Opt. 38, 6237-6246 (1999).

22. E. A. Bucher, "Computer simulation of light pulse propagation for communication through thick clouds," Appl. Opt. 12, 23912400 (1973).

23. A. I. Carswell, "Laser measurements in clouds," in Clouds: Their Formation, Optical Properties and Effects, A. Deepak and P. V. Hobbs, eds. (Academic, New York, 1981), pp. 363406.

24. G. E. Thomas and K. Stamnes, Radiative Transfer in the Atmosphere and Ocean (Cambridge U. Press, Cambridge, UK, 1999).

25. M. C. Jermy and A. Allen, "Simulating the effects of multiple scattering on images of dense sprays and particle fields," Appl. Opt. 41, 4188-4196 (2002).

26. M. C. Jermy, A. Allen, and A. K. Vuorenkoski, "Simulating the effect of multiple scattering on images of dense sprays," in Optical and Laser Diagnostics: Proceedings of the First IOP Conference Series, C. Arcoumanis and K. T. V. Grattan, eds. (Institute of Physics, Bristol, UK, 2003), Vol. 177, pp. 89-94.

27. E. Berrocal, A. Allen, and M. Jermy, "Monte Carlo simulation of laser imaging diagnostics in polydisperse dense sprays," http://optics.sgu.ru/SFM/2003/internet/berrocal/index_files/ frame.htm.

28. V. L. Kuzmin and I. V. Meglinski, "Coherent multiple scattering effects and Monte Carlo method," JETP Lett. 79, 109-112 (2004).

29. I. V. Meglinski, V. L. Kuzmin, D. Y. Churmakov, and D. A.
Greenhalgh, "Monte Carlo simulation of coherent effects in multiple scattering," Proc. R. Soc. London Ser. A 461, 43-53 (2005).

30. S. Bartel and A. H. Hielscher, "Monte Carlo simulations of the diffuse backscattering Mueller matrix for highly scattering media," Appl. Opt. 39, 1580-1588 (2000).

31. X. Wang, L.-H. Wang, C.-W. Sun, and C. C. Yang, "Polarized light propagation through the scattering media: time-resolved Monte Carlo and experiments," J. Biomed. Opt. 8, 608-617 (2003).

32. K. Muinonen, "Coherent backscattering of light by complex random media of spherical scatterers: numerical solution," Waves Random Media 14, 365-388 (2004).

33. S. V. Gangnus, S. J. Matcher, and I. V. Meglinski, "Monte Carlo modeling of polarized light propagation in biological tissues," Laser Phys. 14, 886-891 (2004).

34. T. Iwai, H. Furukawa, and T. Asakura, "Numerical analysis on enhanced backscatterings of light based on Rayleigh-Debye scattering theory," Opt. Rev. 2, 413-419 (1995).

35. A. S. Martinez and R. Maynard, "Polarization statistics in multiple scattering of light: a Monte Carlo approach," in Photonic Band Gaps and Localization, C. M. Souloukis, ed. (Plenum, New York, 1993), pp. 99-114.

36. V. L. Kuzmin, I. V. Meglinski, and D. Y. Churmakov, "Coherent effects under multiple scattering of linearly polarized light," Opt. Spectrosc. 98, 673-679 (2005).

37. H. Ishimoto and K. Masuda, "A Monte Carlo approach for the calculation of polarized light: application to an incident narrow beam,” J. Quant. Spectrosc. Radiat. Transfer 72, 467-483 (2002).

38. M. J. Raković, G. W. Kattawar, M. Mehrübeoğlu, B. D. Cameron, L. V. Wang, S. Rastegar, and G. L. Coté, "Light backscattering polarization patterns from turbid media: theory and experiment," Appl. Opt. 38, 3399-3408 (1999).

39. X. Wang and L. V. Wang, "Propagation of polarized light in birefringent turbid media: a Monte Carlo study," J. Biomed. Opt. 7, 279-290 (2002).

40. S. M. Rytov, Yu. A. Kravtsov, and V. I. Tatarski, Principles of Statistical Radiophysics (Springer, Berlin, 1987).

41. P. M. Chaikin and T. C. Lubensky, Principles of Condensed Matter Physics (Cambridge U. Press, Cambridge, UK, 1995).

42. V. L. Kuzmin, V. P. Romanov, and L. A. Zubkov, "Propagation and scattering of light in fluctuating media," Phys. Rep. 248, 71-368 (1994).

43. G. Mie, "Considerations on the optic of turbid media, especially colloidal metal sols," Ann. Phys. (Leipzig) 25, 377-442 (1908).

44. L. G. Henyey and J. L. Greenstein, "Diffuse radiation in the galaxy," Astrophys. J. 93, 70-83 (1941).

45. J. R. Zijp and J. ten Bosch, "Use of tabulated cumulative density functions to generate pseudorandom numbers obeying specific distributions for Monte Carlo simulations," Appl. Opt. 33, 533-534 (1994).

46. D. Toublanc, "Henyey-Greenstein and Mie phase functions in Monte Carlo radiative transfer computations,” Appl. Opt. 35, 3270-3274 (1996).

47. E. Tilnet, S. Avrillier, and M. Tualle, "Fast semianalytical Monte Carlo simulation for time-resolved light propagation in turbid media,” J. Opt. Soc. Am. A 13, 1903-1915 (1996).

48. L. V. Adzhemyan, L. Ts. Adzhemyan, L. A. Zubkov, and V. P. Romanov, "Molecular light scattering of varying multiplicity," Sov. Phys. JEPT 53, 278-281 (1981).

49. K. K. Bizheva, A. M. Siegel, and D. A. Boas, "Path-length resolved dynamic light scattering in highly scattering random media: the transition to diffusing wave spectroscopy," Phys. Rev. E 58, 7664-7667 (1998). 